\title{
Adaptations in Glutamate and Glycine Content within the Lumbar Spinal Cord Are Associated with the Generation of Novel Gait Patterns in Rats following Neonatal Spinal Cord Transection
}

\author{
Mary Jo Cantoria, Pamela Anne See, Harmit Singh, and Ray D. de Leon \\ School of Kinesiology and Nutritional Science, California State University, Los Angeles, California 90032-8162
}

\begin{abstract}
After spinal cord transection, the generation of stepping depends on neurotransmitter systems entirely contained within the local lumbar spinal cord. Glutamate and glycine likely play important roles, but surprisingly little is known about how the content of these two key neurotransmitters changes to achieve weight-bearing stepping after spinal cord injury. We studied the levels of glutamate and glycine in the lumbar spinal cord of spinally transected rats. Rats $(n=48)$ received spinal cord transection at 5 days of age, and 4 weeks later half were trained to step using a robotic treadmill system and the remaining half were untrained controls. Analyses of glutamate and glycine content via high-performance liquid chromatography showed training significantly raised the levels of both neurotransmitters in the lumbar spinal cord beyond normal. The levels of both neurotransmitters were significantly correlated with the ability to perform independent stepping during training. Glutamate and glycine levels were not significantly different between Untrained and Normal rats or between Trained and Untrained rats. There was a trend for higher expression of VGLUT1 and GLYT2 around motor neurons in Trained versus Untrained rats based on immunohistochemical analyses. Training improved the ability to generate stepping at a range of weight support levels, but normal stepping characteristics were not restored. These findings suggested that the remodeling of the lumbar spinal circuitry in Trained spinally transected rats involved adaptations in the glutamatergic and glycinergic neurotransmitter systems. These adaptations may contribute to the generation of novel gait patterns following complete spinal cord transection.
\end{abstract}

\section{Introduction}

Animals can generate full weight-bearing hindlimb stepping even after a complete transection of the spinal cord. Because spinal transection eliminates serotonergic and noradrenergic descending fibers, other neurotransmitters found within the local lumbar spinal cord circuitry play more significant roles in locomotion (Giroux et al., 2003). Recent findings indicated that training rats that received spinal cord transections as neonates restored a normal balance of excitatory and inhibitory synapses on motor neurons (Ichiyama et al., 2011). These findings suggested that the levels of excitatory and inhibitory neurotransmitters within the lumbar spinal cord circuitry were critical for stepping, but to date only the levels of GABA have been shown to be modulated by locomotor training in adult spinally transected (ST) cats (Tillakaratne et al., 2002). Glutamate and glycine play vital roles within

Received July 4, 2011; revised Oct. 26, 2011; accepted 0ct. 28, 2011.

Author contributions: R.D.d.L. designed research; M.J.C. and P.A.S. performed research; H.S. contributed unpublished reagents/analytic tools; M.J.C. and P.A.S. analyzed data; M.J.C., P.A.S., H.S., and R.D.d.L. wrote the paper.

This work was supported by NIH Grant R01NS055911 and an American Recovery and Reinvestment Act (ARRA) supplement.

The authors declare no competing financial interests.

Correspondence should be addressed to Prof. Ray D. de Leon, School of Kinesiology and Nutritional Science, California State University, Los Angeles, 5151 State University Drive, Physical Education 206, Los Angeles, CA 90032 8162. E-mail: rdeleon@calstatela.edu.

DOI:10.1523/JNEUROSCI.3499-11.2011

Copyright $\odot 2011$ the authors $\quad 0270-6474 / 11 / 3118598-08 \$ 15.00 / 0$ the mammalian lumbar spinal cord circuits that control locomotion (Kiehn, 2006). Glutamate mediates excitatory neurotransmission used for neuronal activation and for rhythm generation, but also for providing excitatory drive to central pattern generator (Kiehn et al., 2008). Glycine mediates inhibitory neurotransmission that is necessary for maintaining interlimb and intralimb coordination (Kiehn, 2006). Based on pharmacological studies, the recovery of stepping in adult ST cats involved changes in both neurotransmitter systems (de Leon et al., 1999; Giroux et al., 2003). However, how the levels of glutamate and glycine in the lumbar spinal cord were changed by training and whether these changes affected weight-bearing stepping have not yet been examined.

In the present study, we examined the effects of weightsupported treadmill training on glutamate and glycine levels in the lumbar spinal cord of rats that received spinal cord transections as neonates. A robotic device was used to train the rats and to test stepping at challenging weight-support levels. We used high-performance liquid chromatography (HPLC) to analyze glutamate and glycine levels within the lumbar spinal cord. HPLC provides a sensitive measurement of the concentration of neurochemicals within nervous tissue and has been used to measure exercise-induced changes in glutamate and glycine levels in the lumbar spinal cord of intact rats (Skup et al., 2007). We also performed immunohistochemical analyses of the glutamate and glycine vesicular transporters, VGLUT1 and GLYT2. Previous 


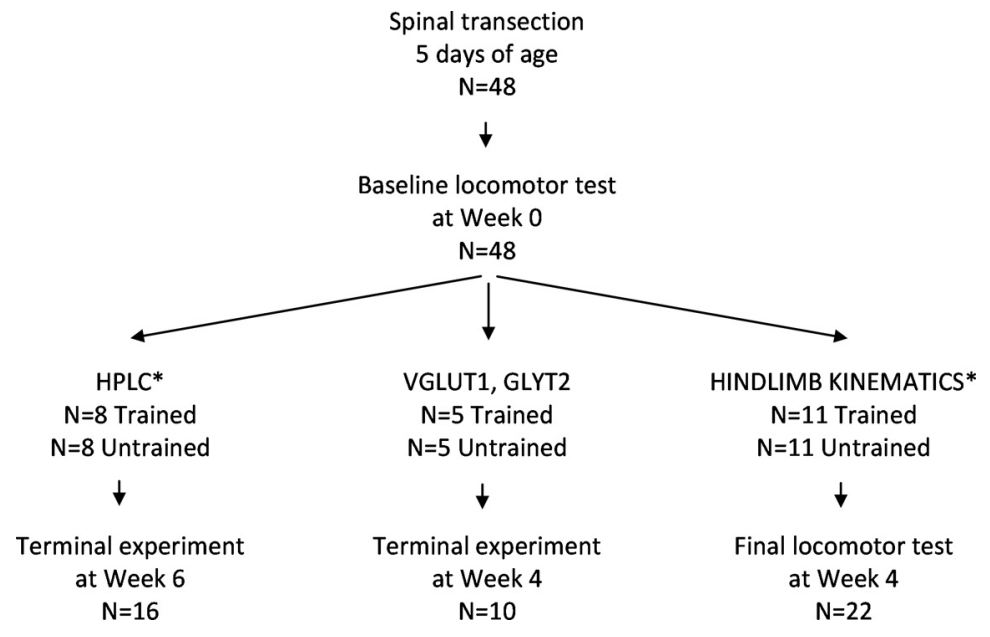

Figure 1. Experimental design (see Materials and Methods). Trained and Untrained rats that received neonatal spinally cord transection were used in three experiments: (1) HPLC to measure glutamate and glycine levels; (2) immunohistochemical experiments for VGLUT1 and GLYT2 expression; and (3) hindlimb kinematic analyses. Asterisk $\left(^{*}\right)$ denotes that Normal rats were also included for the HPLC experiment $(n=4)$ and for the kinematic analyses experiment $(n=9)$.

HPLC studies have reported that glutamate and glycine levels at or near the injury site immediately drop after spinal cord injury (Demediuk et al., 1989; Diaz-Ruiz et al., 2007). Although long-term changes were not examined, these findings suggested that depressed levels of glutamate and glycine may impact the ability to generate stepping. We hypothesized that long-term training would restore normal levels of glutamate and glycine in the lumbar spinal cord, thereby facilitating locomotor recovery. Contrary to this hypothesis, training raised the concentration of both neurotransmitters beyond normal, and the best-stepping trained rats had the greatest glutamate and glycine levels. Training improved stepping but resulted in a pattern of hindlimb locomotion that did not resemble the normal pattern. The present findings suggested that abnormally high levels of glutamate and glycine within the lumbar spinal cord circuitry contributed to novel gait patterns in trained ST rats.

\section{Materials and Methods}

Experimental design. The design of the experiment is described in Figure 1. A total of 48 female Sprague Dawley rats received a complete midthoracic spinal cord transection at $5 \mathrm{~d}$ of age. Four weeks after surgery, baseline locomotor testing was performed using a robotic treadmill system. The occurrence of the baseline test will be referred to as Week 0 . The rats were then distributed into two groups, Trained and Untrained, which were used in three different experiments. To examine the effects of training on glutamate and glycine content in the lumbar spinal cord, Trained $(n=8)$ and Untrained rats $(n=8)$ were killed at Week 6 (i.e., 6 weeks after baseline testing) and their spinal cords were removed and processed for HPLC (see HPLC in Fig. 1). To examine the cellular expression of glycine and glutamate, Trained $(n=5)$ and Untrained $(n=$ 5) rats were killed by perfusion at Week 4 and the spinal cords were removed and processed for immunohistochemistry (see VGLUT1, GLYT2 in Fig. 1). To examine the effects of training on kinematic characteristics of stepping, Trained $(n=11)$ and Untrained $(n=11)$ rats underwent a final locomotor test at Week 4 (see HINDLIMB KINEMATICS in Fig. 1). The rats in the HPLC and immunohistochemistry experiments were not retested before the terminal experiment to avoid any training effects on glutamate and glycine due to testing. However, in the HPLC experiment, locomotor performance of the Trained rats was scored during each training session. Data from additional intact rats were included for comparisons to normal stepping $(n=9)$ and normal levels of glutamate and glycine $(n=4)$. All procedures were performed in accordance with the Institutional Animal Care and Use Committee at California State University, Los Angeles, CA.

Spinal cord transection procedure. Spinal cord transection procedures were performed under aseptic conditions as described previously (Cha et al., 2007). Briefly, 5-d-old pups were anesthetized using an isoflurane gas chamber (VetEquip V-10 Mobile). A skin incision was made over the midthoracic vertebrae, and the overlying fascia and muscles were retracted to expose the dorsal surface of the vertebrae. A partial laminectomy was performed at the midthoracic level, and then the spinal cord was lifted gently using fine forceps and completely transected using microscissors. Small cotton balls were used to produce a 2-3 $\mathrm{mm}$ cavity and to verify the completeness of the transection. Gel foam was inserted into the cavity and the incision was closed using 5.0 Ethilon suture. After surgery, the rat pups were allowed to fully recover in a warm incubator $\left(80^{\circ} \mathrm{C}\right)$ and regain consciousness before being returned to their respective dams.

The pups were weaned 3 weeks postsurgery and each cage housed two to three pups under standard conditions of humidity, temperature, and $12 \mathrm{~h}$ light/dark cycle. After weaning, the rats were weighed daily and the bladders and colons were monitored twice a day. The rats had access to food and water ad libitum.

Robotic treadmill training, testing, and analyses. A detailed description of the robotic device (Rodent Robot 3000, Robomedica) has been reported previously (Cha et al., 2007). In short, the device is comprised of two lightweight robotic arms, a body weight support system, and a variable speed treadmill. The robotic arms were secured around the hindlimb via neoprene cuffs wrapped around the ankles. The amount of load imposed on the hindlimbs was adjusted through the computer-controlled body weight support system to provide $75-95 \%$ weight support, and the speed of walking was set at $8 \mathrm{~cm} \cdot \mathrm{s}^{-1}$. During training, customized software was used to count the number of steps. A step was defined as ankle movements that displaced the robotic arm by $\geq 10 \mathrm{~mm}$ in the forward, horizontal direction. The number of steps was displayed on a computer monitor in real time, and a training session was completed once the rats reached 1000 steps (total combined for both left and right hindlimbs). Typically, the duration of training episodes was $\sim 20 \mathrm{~min}$. If the rats were unable to perform independent stepping, the trainers provided manual assistance to move the hindlimbs. To assess the ability to perform independent stepping during training, a three-point scoring system was used. An inability to step without assistance throughout the training session received a 0 score. Independent stepping for less than half of the required 1000 steps received a score of 1 . Independent stepping for greater than half of the 1000 steps received a score of 2. Independent stepping for all of the 1000 steps received a score of 3 .

During testing, the ability to perform unassisted bipedal, hindlimb stepping on the treadmill $(8 \mathrm{~cm} \cdot \mathrm{s}-1)$ was assessed at 95,85 , and $75 \%$ body weight support levels. An $85 \%$ weight support level was an optimal level for stepping, whereas 95 and $75 \%$ are high and low amounts of weight support, respectively, that challenge the ability of the ST rats to generate stepping (Timoszyk et al., 2005). Data were also collected from Normal rats after the rats were acclimated to stepping in the robotic system at $85 \%$ weight support. Ankle movements were recorded by the robotic device for $30 \mathrm{~s}$ at each weight support and the data were stored on a computer. The trainers did not assist stepping during testing. If the rats were unable to perform stepping and the hindlimbs dragged for $10 \mathrm{~s}$, the trainers repositioned the rats by lifting the rats and placing their hindpaws on the treadmill. Analyses of ankle position were performed as described previously (Cha et al., 2007). Briefly, the start and end for each locomotor movement were detected based on horizontal and vertical velocities of the robotic arm. This allowed for comprehensive analyses of 
locomotor movements during testing (i.e., 2436 and 2818 total locomotor movements in the Untrained and Trained rats, respectively). Step height was defined as the distance between the maximum and minimum vertical positions of the ankle during a step. Step length was defined as the horizontal distance traveled during forward ankle movement. Swing and stance duration were the intervals of time during forward and backward movement, respectively. Measurement of the height of the ankle during swing was used to distinguish weight-bearing steps from nonweightbearing (i.e., dragging) steps. Based on our previous studies (Heng and de Leon, 2009), steps in which the ankle was raised $>10 \mathrm{~mm}$ were defined as weight-bearing steps. The percentage of weight-bearing steps was calculated by dividing the number of weight-bearing steps by the total number of locomotor movements detected by the robotic arms $(\times 100 \%)$. The coordination of left and right hindlimb movements was measured as described previously (de Leon et al., 2004). The amount of time that the left and right ankles moved in an alternating pattern during $30 \mathrm{~s}$ of testing was calculated based on the direction of velocities during stepping. Alternating movements were defined as left and right ankle velocities that were opposite in direction.

HPLC analysis. The rats were anesthetized using isoflurane, the head was decapitated, and the brain and lumbar spinal cord were immediately dissected and weighed. For the Trained rats, the terminal experiment occurred within $2 \mathrm{~h}$ after the completion of the last training (Skup et al., 2007). This time frame was chosen to avoid acute effects due to training on amino acid levels (Walwyn et al., 1999). Ice was used to keep the tissues cool during dissection. The tissues were immediately homogenized in $10 \times$ ice-cold $85 \%$ methanol to deproteinate and inactivate the protein (Diaz-Ruiz et al., 2007). Samples were centrifuged at $5000 \times g$ for $15 \mathrm{~min}$ at $4^{\circ} \mathrm{C}$ and supernatants were stored at $-80^{\circ} \mathrm{C}$ until ready for chromatographic analysis. Samples were resolved on a Zorbax Eclipse AAA $4.6 \times 150 \mathrm{~mm}, 5 \mu \mathrm{m}$ column (Agilent Technologies) using the manufacturer's instructions for offline precolumn derivatization. A 2.5 $\mu$ lvolume of borate buffer $(0.4 \mathrm{~N}, \mathrm{pH} 10.2)$ and $0.5 \mu \mathrm{l}$ of the sample were mixed and allowed to react for half a minute and then derivatized by 0.5 $\mu \mathrm{l}$ of ortho-phthalaldehyde (OPA). After mixing, $32 \mu \mathrm{l}$ of water was added and the solution was mixed before $0.5 \mu \mathrm{l}$ aliquots were injected into the stationary phase. A binary mobile phase of (A) $40 \mathrm{~mm}$ sodium

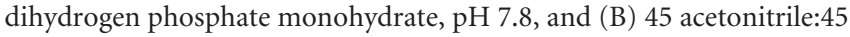
methanol: 10 water was used under linear gradient conditions (Solvent B: $57 \%$ at $18.1 \mathrm{~min}, 100 \%$ at $18.6-22.3 \mathrm{~min}$ and $0 \%$ at $23.2-26 \mathrm{~min}$ ). Chromatographic analyses were carried out using Agilent Technologies 1100 HPLC system in quadruplicate. Pure glutamate and glycine were used as external standards measured in triplicate. Reagent grade amino acid standards and sodium dihydrogen phosphate monohydrate were purchased from Sigma-Aldrich. HPLC grade acetonitrile, methanol, and water were obtained from Fisher Scientific, while HPLC grade borate buffer and OPA were procured from Agilent Technologies.

Immunohistochemical experiments. One day after the final training session, the ST rats were anesthetized with isoflurane and then underwent transcardial perfusion ( $4 \%$ paraformaldehyde), and the spinal cords were dissected, blocked, and cut into $30 \mu \mathrm{m}$ sections as described previously(Tillakaratne et al., 2002). Two immunohistochemical experiments were performed. One experiment was performed with VGLUT1 and HSP27 antibodies and another experiment with GLYT2 and HSP27 antibodies. HSP27 was used to label motor neurons in the ventral horn (Plumier et al., 1997). For each experiment, the tissue sections from all groups were processed simultaneously in a single experiment. The conditions for all procedures were identical for all the sections in every group. The spinal cord sections from the L2-L5 region ( $3-5$ sections/rat) were washed and then transferred to a blocking solution of 3\% normal donkey serum (NDS). Spinal cord sections were then transferred into 96-well plates and incubated overnight with one primary antibody (i.e., guinea pig VGLUT1 antibody at 1:1000 or guinea pig GLYT2 antibody at $1: 1000$ ) followed by an overnight incubation with the other primary antibody (goat HSP27 antibody at 1:500). Afterward, the spinal sections were washed, blocked in 3\% NDS, and then incubated for $1 \mathrm{~h}$ in anti-goat secondary antibody (rhodamine at 1:200) and anti-guinea pig secondary antibody (FITC at 1:200). The spinal cord sections were transferred to glass slides, air dried, and coverslipped with VectaShield (Vector Laboratories) with DAPI staining.

Microscopic imaging and analyses. For analyses of labeling, microscopic images were acquired using an Olympus FluoViewTM FV500 confocal laser scanning microscope (100× magnification). All images were acquired on one day under the same conditions and settings. Images of HSP27-positive motor neurons in Lamina IX were acquired. Six optical slices ( $5 \mu \mathrm{m}$ thick) were scanned through each motor neuron. We determined from pilot experiments that $5 \mu \mathrm{m}$ thickness was ideal. VGLUT1 and GLYT2 labeling were found primarily on the perimeter of motor neurons. Simple PCI 6 software (Compix) was used to analyze VGLUT1 and GLYT2 labeling based on a technique developed by Macias et al. for analyzing labeling around motor neurons (Macias et al., 2009). Using the closed polygon object drawing tool, an outline was generated that extended just beyond the perimeter of HSP27-labeled motor neurons. The area of VGLUT1 and GLYT2 labeling $2 \mu \mathrm{m}$ beyond and $2 \mu \mathrm{m}$ inside the perimeter of the motor neuron was measured after background signal was eliminated (based on a control section with secondary antibody only). These areas were then normalized to motor neuron area (VGLUT1 or GLYT2 area/motor neuron area). All analyses were performed using the Simple PCI 6 software (Compix).

Data analysis. One-way ANOVA was used to analyze all differences between the Trained, Untrained, and Normal groups. Post hoc comparisons between pairs of groups was performed using Tukey test. Correlations between amino acid content and stepping performance during training were analyzed using Pearson's correlation with significance test. Due to variability in day-to-day performance, the average locomotor performance during the entire 6 weeks of training was calculated for these correlations instead of using performance data from a single day. Kinematic measurements that were expressed as a percentage of normal values were statistically compared to normal using a one-sample $t$ test. All statistical analyses were performed using SPSS.

\section{Results}

Abnormally high glutamate and glycine levels in the lumbar spinal cord were correlated with the ability to perform independent stepping

Based on HPLC, the concentration of glutamate and glycine in the lumbar spinal cord was significantly greater in the Trained ST rats relative to Normal (compare Trained to Normal in Fig. 2A). The concentrations in the Untrained rats was not significantly different from those in Normal rats (compare Untrained to Normal in Fig. 2A). The concentration of glutamate and glycine in the lumbar spinal cord was not significantly different between the Trained and Untrained rats (compare Trained to Untrained in Fig. 2A).

The average locomotor scores over the 6 weeks of training were calculated for each Trained rat (Table 1). The best rats were capable of independent stepping for at least half of the training session (see R6, R17, R29, and R26 in Table 1), whereas the remaining rats required assistance from the trainers for most of the training session (see R37, R36, R1, and R34 in Table 1). Glutamate and glycine levels were positively correlated with the average locomotor score, and these correlations were highly significant (Fig. $2 B, C$; test of significance for Pearson's correlation coefficient, $p<0.01$ ). The greatest levels of glutamate and glycine were found in the best performing rats whereas the worst performing rats had the lowest levels of glutamate and glycine (Fig. 2B,C). Unlike the lumbar spinal cord, the cortical levels of glutamate and glycine were not correlated with locomotor scores (Fig. 2D).

\section{Expression of VGLUT1 and GLYT2 around motor neurons in trained and untrained rats}

VGLUT1 and GLYT2 labeling was found throughout the ventral horn in the lumbar spinal cord as described previously (Kitzman, 
A

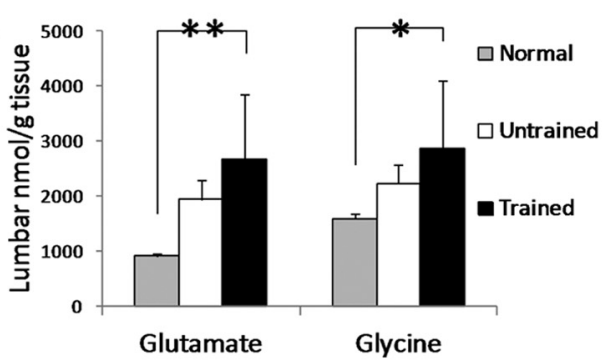

Glutamate

Glycine

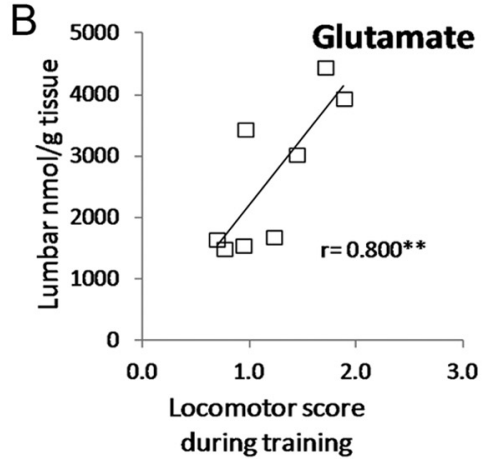

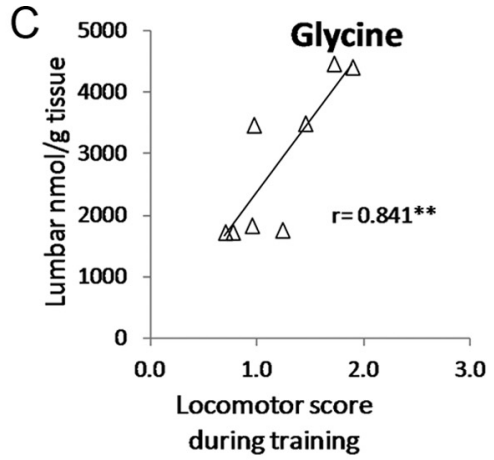

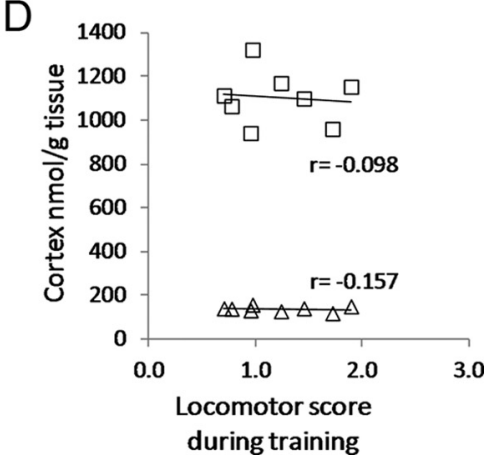

Figure 2. HPLC-measured glutamate and glycine levels in the lumbar spinal cord. $A$, Glutamate and glycine levels in Normal (gray), Untrained ST rats (white) and Trained ST rats (black). Asterisks ** and ${ }^{*}$ indicate significant difference from Normal at $p<$ 0.01 and $p<0.05$ levels, respectively. $\boldsymbol{B}, \boldsymbol{C}$, The correlation of glutamate $(\boldsymbol{B})$ and glycine $(\boldsymbol{C})$ levels in the lumbar spinal cord and locomotor score during training (see Table 1 for details) are shown. $\boldsymbol{D}$, Correlation of glutamate (squares) and glycine (triangles) levels in the cortex and locomotor score during training. Pearson correlation coefficients are shown on each plot in $\boldsymbol{B}, \boldsymbol{C}$, and $\boldsymbol{D}$. Asterisks ${ }^{* *}$ indicate significant correlation at $p<0.01$ level.

Table 1. Average locomotor scores during six weeks of training in ST rats

\begin{tabular}{lc}
\hline Rat number & Average locomotor score (mean $\pm \mathrm{SE}$ ) \\
\hline R6 & $1.9 \pm 0.1$ \\
R17 & $1.7 \pm 0.2$ \\
R29 & $1.5 \pm 0.1$ \\
R26 & $1.2 \pm 0.1$ \\
R37 & $0.96 \pm 0.1$ \\
R36 & $0.95 \pm 0.1$ \\
R1 & $0.77 \pm 0.2$ \\
R34 & $0.69 \pm 0.1$ \\
\hline
\end{tabular}

Locomotor performance during daily training sessions was scored using a $0-3$ scale that assessed their ability to perform independent stepping without assistance by the trainers to move the hindlimbs: 0 , № independent stepping; 1 , independent stepping less than half of the 1000 steps; 2 , independent stepping greater than half of the 1000 steps; 3 , independent stepping for all of 1000 steps. The average locomotor score was calculated over 30 training sessions ( 5 sessions per week for 6 weeks) for each rat.

2007; Landry et al., 2004) (Fig. 3 A,D). VGLUT1 and GLYT2 labeling was found around HSP27-positive motor neurons in both the Untrained (Fig. 3B,E) and Trained rats (Fig. 3C,F). Quantitative analysis was performed to compare the amounts of VGLUT1 and GLYT2 labeling around HSP27-labeled motor neurons. The average number of motor neurons that was analyzed was not significantly different between the Trained and Untrained groups (e.g., Trained, $50.0 \pm 11.1$; Untrained, $49.8 \pm 6.2$ ). The average area of VGLUT1 labeling relative to motor neuron area (VGLUT1 area/motor neuron area) was $0.08 \pm 0.03$ in the Untrained group and $0.14 \pm$ 0.02 in the Trained rats group. This difference approached statistical significance $(p=0.07)$. The average area of GLYT2 labeling relative to motor neuron area (GLYT2 area/motor neuron area) was $0.02 \pm$ 0.01 and $0.16 \pm 0.04$ in Untrained and Trained rats, respectively. This difference also approached statistical significance $(p=0.06)$.
Training improved stepping but did not restore normal stepping characteristics The robotic device recorded ankle trajectory during stepping (see Fig. 4A, C, Ankle trajectory inset) and differentiated weightbearing from nonweight-bearing locomotor movements (Fig. 4A, C, Ankle horizontal displacement, arrows). The total number of locomotor movements (weight bearing plus nonweight bearing) was not significantly different between the Trained and Untrained rats at Week 0 (Untrained, $120 \pm 12$; Trained, $139 \pm 18$ ) or Week 4 (Untrained, $111 \pm 21$; Trained: $117 \pm 14$ ). The Trained rats performed a significantly greater percentage of weight-bearing steps than the Untrained rats at Week 4 during testing at the $95 \%$ and $75 \%$ weight support levels (Fig. 4D, compare Untrained versus Trained, 95\% and 75\%). The gap in stepping was largest at the $95 \%$ weight support level where the Trained rats performed twice as many steps as the Untrained rats (Fig. 4D, compare Untrained versus Trained, 95\%). No difference in weightbearing steps was found during baseline testing (Fig. 4B, compare Untrained vs Trained). Overall, a significantly smaller percentage of weight-bearing steps was performed at $95 \%$ than the other weight support levels (Fig. 4B,D, compare 95\% with $85 \%$ and $75 \%$ ).

We compared stepping characteristics in the Untrained and Trained ST rats to stepping in Normal rats that had been acclimated to stepping at $85 \%$ weight support in the robotic system (see Fig. 5). At Week 0 and Week 4, the ankle in the ST rats moved along an elliptical trajectory that did not follow the Normal ankle trajectory patterns (Fig. 5A, B, compare thick and thin lines with dashed line). At Week 4 , the ankle trajectory in the Trained rats was larger than the one in the Untrained rats (Fig. $5 B$, compare thick and thin lines), and this was supported by the kinematic analyses (Fig. B, see Area and Length). Cycle period was not significantly different from Normal at Week 0 and Week 4 (Fig. $5 A, B$, see $\mathrm{CP}$ ). No differences in swing and stance duration were found (data not shown).

To assess the coordination of the left and right hindlimb movements, the amount of time that the ankles moved in an alternating pattern (i.e., opposite directions) instead of a synchronous pattern (i.e., same direction) was calculated. The ST rats performed less alternating movements than Normal rats (Fig. 5A, $B$, see Lt-Rt). At Week 0, no difference was found between Trained and Untrained rats (Fig. 5A, Lt-Rt). At Week 4, the amount of time in which alternating movements occurred in the Trained rats was significantly greater than that in the Untrained rats (Fig. 5B, see Lt-Rt).

\section{Discussion}

To summarize, we examined the effects of treadmill training in rats that received spinal cord transections as neonates. Training increased the amount of glutamate and glycine in the lumbar spinal cord beyond normal levels. The levels of these two key neurotransmitters were significantly correlated with stepping performance during training. There was a trend for greater expres- 

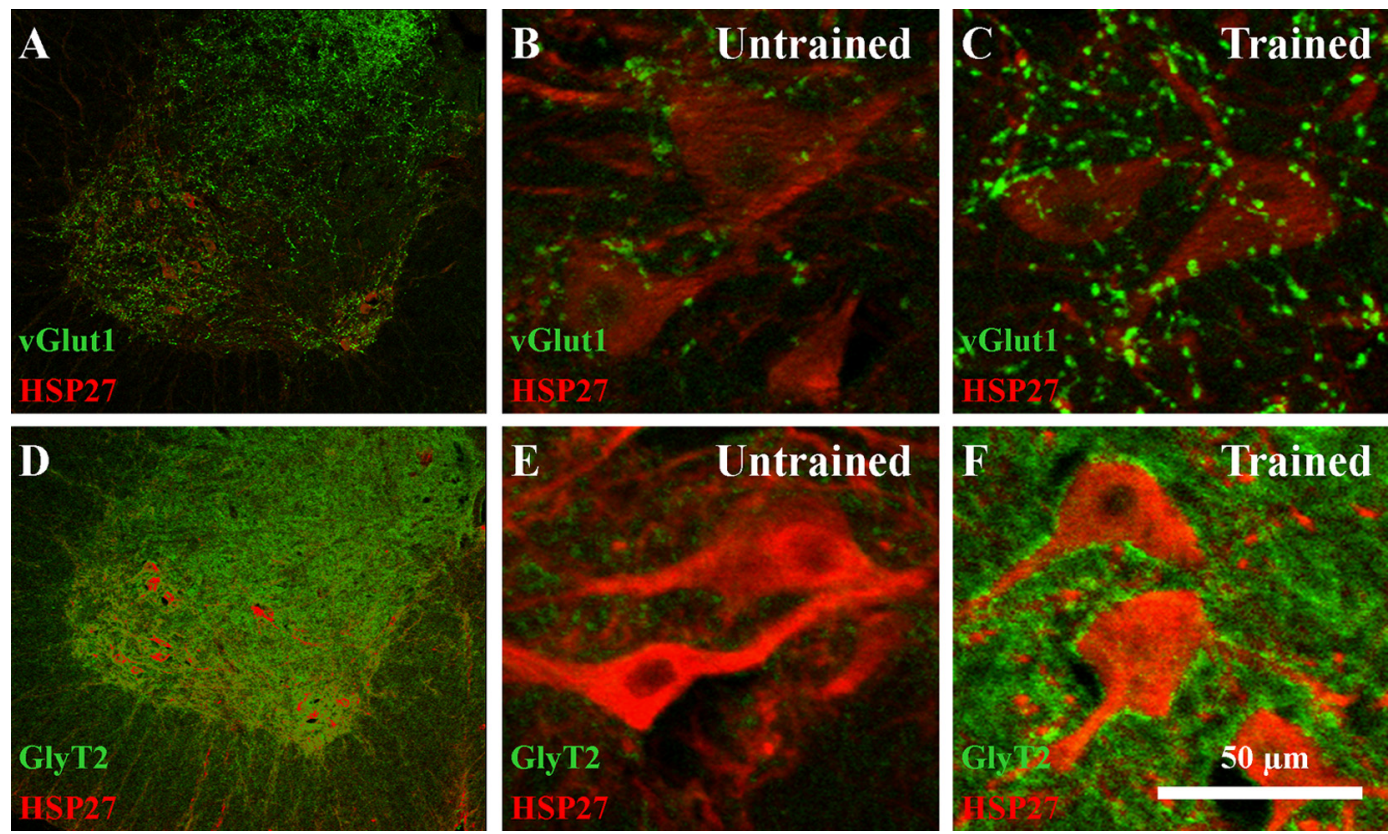

Figure 3. Expression of VGLUT1 and GLYT2 in lumbar spinal cord. $A$, VGLUT1 (green) expression in the ventral horn of the lumbar spinal cord in a Trained rat. Motor neurons were labeled with HSP27 (red). B, C, VGLUT1 expression (green) around HSP27-labeled motor neurons is shown in a representative Untrained ( $\boldsymbol{B}$ ) and Trained (C) ST rat, respectively. D, GLYT2 (green) expression in the ventral horn of the lumbar spinal cord in a Trained rat. Motor neurons were labeled with HSP27 (red). E, F, GLYT2 expression (green) around HSP27-labeled motor neurons is shown in a representative Untrained $(\boldsymbol{E})$ and Trained $(\boldsymbol{F})$ ST rat respectively.

A

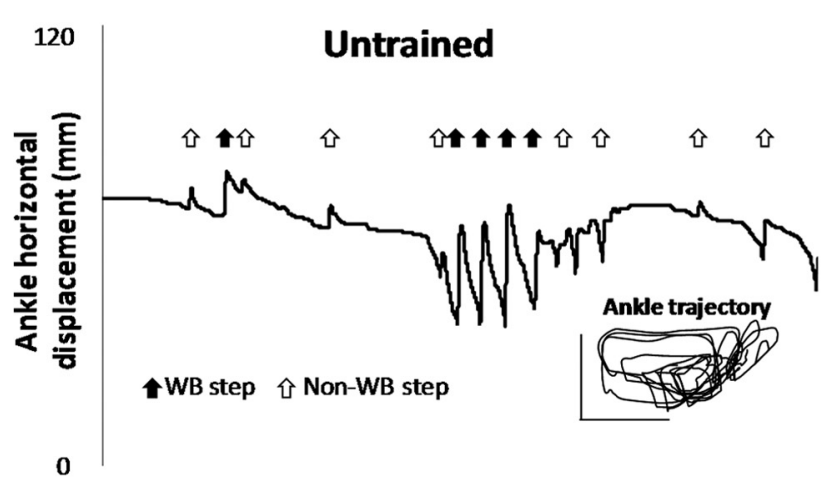

B

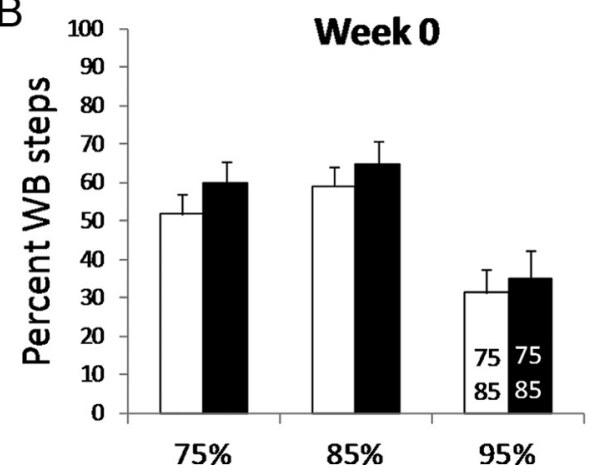

C
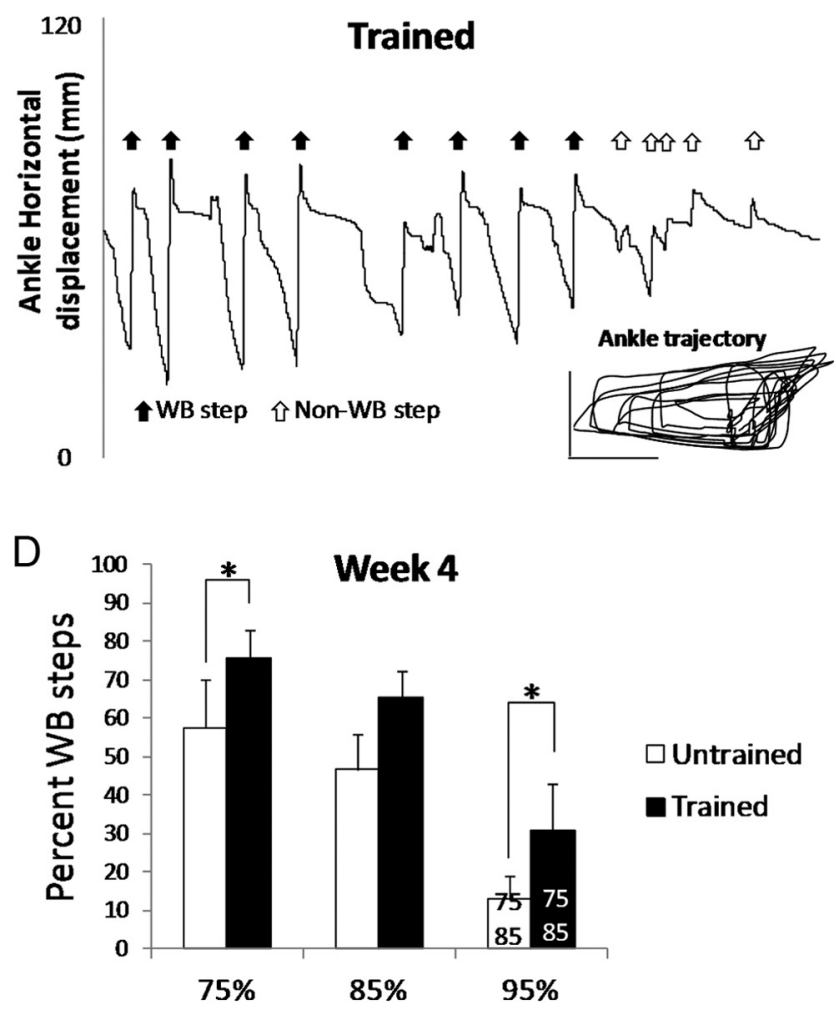

Figure 4. Comparison of hindlimb treadmill stepping in Untrained and Trained ST rats. $\boldsymbol{A}, \boldsymbol{C}$, Horizontal ankle movements recorded by the robotic device and the corresponding ankle trajectory are shown for representative Untrained $(\boldsymbol{A})$ and Trained $(\boldsymbol{C})$ rats. Arrows in the horizontal ankle movement indicate forward movements detected by the robotic device. Black arrows indicate weight-bearing steps whereas white arrows indicate nonweight-bearing movements. The vertical and horizontal calibration bars in the ankle trajectory are both 20 mm. The data were collected during 30 s of testing at $95 \%$ weight support at Week 4 . $\boldsymbol{B}, \boldsymbol{D}$, The percentage of steps that were weight bearing (WB) during testing at Week 0 ( $\boldsymbol{B}$ ) and Week 4 $(\boldsymbol{D})$ is shown for Untrained (white) and Trained (black) rats. The data are from tests at $95 \%, 85 \%$, and $75 \%$ weight support levels. The data shown are averages ( $n=11$ for each group). Asterisks $\left({ }^{*}\right)$ indicates significant difference between Trained and Untrained ( $p<0.05$ ). The numbers 75 and 85 on the $95 \%$ bar indicate significant difference between $95 \%$ and the $75 \%$ and $85 \%$ body weight support levels, respectively $(p<0.05)$. 
A

Week 0 Average Ankle Trajectory

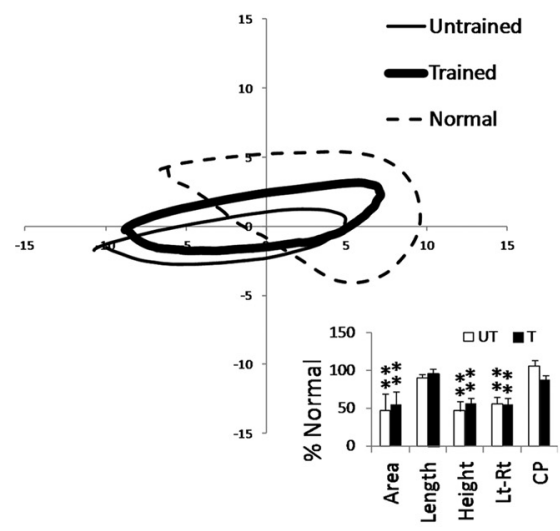

B

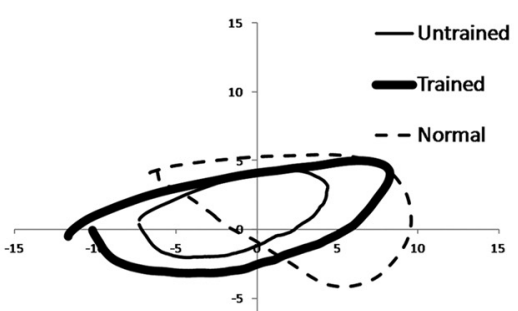

Figure 5. Average ankle trajectories during hindlimb treadmill stepping in Normal and ST rats. $A, B$, The average trajectory of the ankle for the Untrained (thin line) and Trained (thick line) ST rats is shown for Week $0(\boldsymbol{A})$ and Week $4(\boldsymbol{B})$, respectively. Normal ankle trajectory is also shown (dashed line). The average trajectory for each group was calculated from 11 Untrained, 11 Trained, and 9 Normal rats (during $30 \mathrm{~s}$ of stepping at $85 \%$ weight support for each rat). Bar plots comparing kinematic characteristics between Untrained (UT, white) and Trained (T, black) are shown. Averages are shown ( $n=11$ Untrained; $n=11$ Trained) and are expressed as a percentage of Normal values (100\%). Area, Length, and Height of step cycle trajectory are shown. Lt-Rt is a measure of the alternating movements between the left (Lt) and right (Rt) hindlimbs. CP is cycle period. The asterisks ** and * indicate significant difference from Normal at the $p<0.01$ and $p<0.05$ levels, respectively. Significant differences between Untrained and Trained groups are indicated by the lines and asterisks $\left(^{*}\right)$ above the bars $(p<0.05)$.

sion of the glutamate and glycine vesicular transporters, VGLUT1 and GLYT2, around motor neurons in the Trained versus Untrained rats. The ability to generate alternating, hindlimb stepping at a range of weight support levels was improved by training, but training did not restore normal characteristics of stepping.

\section{Role of glutamate in initiating locomotion after spinal cord transection}

Spinal cord transection results in a loss of nearly all monoaminergic neurotransmitter content in the lumbar spinal cord (Magnusson, 1973). To generate stepping, the lumbar spinal cord circuitry must adapt to the loss of the primary neurotransmitters responsible for activating the locomotor generating circuitry. For example, recent evidence indicates remarkable changes in serotonergic receptor function after transection in adult rats such that these receptors can impact locomotion even in the absence of neurotransmitter (Murray et al., 2010), and a similar phenomenon may occur in noradrenergic receptors (Rank et al., 2011). Compensatory changes in neurotransmitter systems contained within the spinal circuitry likely also occur, but this has only been demonstrated for the inhibitory neurotransmitter, GABA, in adult ST cats (Tillakaratne et al., 2002). Glutamate is the major excitatory neurotransmitter used by interneurons in the lumbar spinal cord circuitry. It is well known that glutamate is essential for intrinsic rhythm generating capability, but it also functions in the initiation of locomotion by providing extrinsic drive to the central pattern generator (Kiehn, 2006). Although no other studies have measured glutamate levels in trained ST animals, findings from previous studies suggested a change in the "pharmacology of locomotion" after spinal cord transection in adult cats (Giroux et al., 2003). Administration of the glutamate receptor antagonist, APV (DL-2-amino-5-phosphonovalerate) completely abolished locomotion in spinal cats $(>4$ weeks postspinal) but did not prevent stepping in normal cats (Giroux et al., 2003). Assuming glutamate receptor expression was unchanged after spinal cord transection (Krenz and Weaver, 1998), the abnormally high levels of glutamate were consistent with the development of an enhanced glutamatergic drive. It is interesting to note that in intact rats, treadmill training did not raise HPLC-measured glutamate levels in the lumbar spinal cord (Skup et al., 2007). Thus, it appeared that spinally transected animals primarily relied on glutamatergic excitation to drive the central pattern generator (CPG), whereas normal animals used pathways involving multiple neurotransmitter systems.

The initiation of locomotion in spinal animals was dependent on sensory afferents from hindlimbs (Rossignol et al., 2008; Courtine et al., 2009; Norton and Mushahwar, 2010). Consistent with these findings, Trained rats in the present study became more responsive to load-related afferent feedback and were better able to use this sensory information to generate successful stepping. Although not significant, there was a trend for VGLUT1, which specifically labels primary afferent terminals (Alvarez et al., 2004), to increase around motor neurons in the ventral horn. Together, these findings suggested that an increase in sensory terminals containing glutamate enhanced sensory drive to lumbar spinal cord neurons that were involved in locomotion. This conclusion is consistent with reported enhanced transmission at synapses between sensory neurons and motor neurons in adult ST rats (Lavrov et al., 2006; Côté et al., 2011) and in neonatal ST rats (Petruska et al., 2007).

Another possibility was that a rise in glutamate levels occurred within interneurons that comprised the central pattern generator. Our analyses of VGLUT1 expression did not include interneurons; thus, we can only speculate on whether increased glutamate facilitated rhythm generation. Two findings in the Untrained rats were relevant here. First, the Untrained rats were capable of producing rhythmic hindlimb locomotor movements on the treadmill. Indeed, the number of locomotor movements (weight bearing plus nonweight bearing) performed by the Trained and Untrained rats was statistically similar. Second, the lumbar spinal cords in the Untrained rats contained normal concentrations of glutamate. These findings supported the conclusion that normal levels of glutamate were sufficient for central pattern generator function in the ST rat. Further studies, however, will be necessary to determine whether the effect of training was to enhance glutamate synapses that were critical for rhythm generation.

\section{Training-enhanced glycinergic inhibition after spinal cord transection}

The increased glycine levels suggested that training enhanced glycinergic inhibition and that this adaptation was beneficial for successful weight-bearing stepping. This finding was not consistent with previous findings suggesting that glycinergic and GABAergic inhibition decreased in trained adult ST cats (de Leon et al., 1999; Tillakaratne et al., 2002). The discrepancy may be related to differences in the age of spinal transection in our study versus previous studies. It is well known that neonatal spinally transected animals recovered better hindlimb function than the adult transected animals (Weber and Stelzner, 1977). After spinal cord transection in adults, inhibition in the lumbar spinal circuits 
increased above normal, and this contributed to an overall depression of hindlimb movements (Robinson and Goldberger, 1986). In contrast, the level of excitation within the lumbar spinal circuitry rose after a spinal cord transection was performed in neonatal rats (Norreel et al., 2003). This was accompanied by a change in hindlimb coordination from the normal left-right alternating pattern to more synchronous activity (Norreel et al., 2003). Locomotor training, then, may have a differential effect on the spinal circuitry of the adult versus the neonatal transected animal. Unlike the adult transected animal, training in neonatal transected animals increased glycine levels, perhaps limiting the enhanced excitability within the lumbar spinal cord circuitry. The increased glycine concentration and improved hindlimb coordination observed in the Trained rats was consistent with this interpretation.

On the other hand, a recent study in neonatally spinally transected rats found that treadmill training reduced inhibitory inputs to motor neurons (Ichiyama et al., 2011). In this study, ultrastructural analyses of terminals were used to identify the inhibitory inputs. This would appear to contradict the VGLUT1 and GLYT2 data in the present study, which suggested that both excitatory and inhibitory inputs were enhanced. However, it is difficult to draw any concrete conclusions regarding the net effect of these VGLUT1 and GLYT2 inputs on motor neuron activity levels. VGLUT1 and GLYT2 only provide an indirect measure of glutamate and glycine neurotransmitters. Other studies have reported that training increased the overall amount of inputs to motor neurons in ST rats, but whether these inputs were excitatory or inhibitory was unknown (Macias et al., 2009; de Leon et al., 2011). The expression of various neurotransmitter receptors on the motor neuron membrane will also be a critical factor in determining overall activity, but little is known about how receptors are modulated in ST animals by treadmill training (Khristy et al., 2009). At this point, the bulk of the data indicates that the effects of training were to increase the excitability of motor neurons, a conclusion that is supported by electrophysiological studies (Petruska et al., 2007; Côté et al., 2011). Clearly, further studies are necessary to understand the roles that the various neurotransmitters, glutamate, glycine, and GABA, and their respective receptors play in shaping motor neuronal activity. It will also be important to continue examining the unique responses to injury in the neonatal nervous system. The neurochemical mechanisms that facilitate functional recovery are likely to be different after a neonatal injury than in adults. This has important ramifications for emerging locomotor therapies that are focused on pediatric spinal cord injured patients (Behrman et al., 2008).

\section{Remodeled spinal circuitry for locomotion}

The locomotor generating circuitry within the lumbar spinal circuitry is profoundly changed after a complete spinal cord transection. Altered physiological, anatomical, and biochemical properties contribute to a new synaptic milieu (Edgerton et al., 1997), as some connections (i.e., supraspinal) are lost and other, remaining connections adapt (Rossignol et al., 2004). The present findings support these ideas, suggesting that abnormal levels of glutamate and glycine developed within the lumbar spinal cord and contributed to learning novel gait patterns. What emerged from all of these changes was a remodeled circuitry capable of accomplishing locomotion even in the absence of connections to the brain (Rossignol et al., 2008; Courtine et al., 2009; Norton and Mushahwar, 2010). Given the remarkable ability of the spinal cord to adapt, it will be important from a clinical standpoint to identify which changes, neurochemical and otherwise, will have the greatest benefit for recovery. This may lead to more effective locomotor training strategies, the development of new strategies, or perhaps combinations of therapies that stimulate plasticity required for stepping.

\section{References}

Alvarez FJ, Villalba RM, Zerda R, Schneider SP (2004) Vesicular glutamate transporters in the spinal cord, with special reference to sensory primary afferent synapses. J Comp Neurol 472:257-280.

Behrman AL, Nair PM, Bowden MG, Dauser RC, Herget BR, Martin JB, Phadke CP, Reier PJ, Senesac CR, Thompson FJ, Howland DR (2008) Locomotor training restores walking in a nonambulatory child with chronic, severe, incomplete cervical spinal cord injury. Phys Ther 88:580-590.

Cha J, Heng C, Reinkensmeyer DJ, Roy RR, Edgerton VR, de Leon RD (2007) Locomotor ability in spinal rats is dependent on the amount of activity imposed on the hindlimbs during treadmill training. J Neurotrauma 24:1000-1012.

Côté MP, Azzam GA, Lemay MA, Zhukareva V, Houlé JD (2011) Activitydependent increase in neurotrophic factors is associated with an enhanced modulation of spinal reflexes after spinal cord injury. J Neurotrauma 28:299-309.

Courtine G, Gerasimenko Y, van den Brand R, Yew A, Musienko P, Zhong H, Song B, Ao Y, Ichiyama RM, Lavrov I, Roy RR, Sofroniew MV, Edgerton VR (2009) Transformation of nonfunctional spinal circuits into functional states after the loss of brain input. Nat Neurosci 12:1333-1342.

de Leon RD, Tamaki H, Hodgson JA, Roy RR, Edgerton VR (1999) Hindlimb locomotor and postural training modulates glycinergic inhibition in the spinal cord of the adult spinal cat. J Neurophysiol 82:359-369.

de Leon RD, Roy RR, Gomez-Pinilla F, Motamedinia P, Edgerton VR (2004) Interlimb coordination after a thoracic hemisection in rats. Basic Appl Myol 14:215-222.

de Leon RD, See PA, Chow CH (2011) Differential effects of low versus high amounts of weight supported treadmill training in spinally transected rats. J Neurotrauma 28:1021-1033.

Demediuk P, Daly MP, Faden AI (1989) Effect of impact trauma on neurotransmitter and nonneurotransmitter amino acids in rat spinal cord. J Neurochem 52:1529-1536.

Diaz-Ruiz A, Salgado-Ceballos H, Montes S, Maldonado V, Tristan L, Alcaraz-Zubeldia M, Ríos C (2007) Acute alterations of glutamate, glutamine, GABA, and other amino acids after spinal cord contusion in rats. Neurochem Res 32:57-63.

Edgerton VR, de Leon RD, Tillakaratne N, Recktenwald MR, Hodgson JA, Roy RR (1997) Use-dependent plasticity in spinal stepping and standing. Adv Neurol 72:233-247.

Giroux N, Chau C, Barbeau H, Reader TA, Rossignol S (2003) Effects of intrathecal glutamatergic drugs on locomotion. II. NMDA and AP-5 in intact and late spinal cats. J Neurophysiol 90:1027-1045.

Heng C, de Leon RD (2009) Treadmill training enhances the recovery of normal stepping patterns in spinal cord contused rats. Exp Neurol 216:139-147.

Ichiyama RM, Broman J, Roy RR, Zhong H, Edgerton VR, Havton LA (2011) Locomotor training maintains normal inhibitory influence on both alpha- and gamma-motoneurons after neonatal spinal cord transection. J Neurosci 31:26-33.

Khristy W, Ali NJ, Bravo AB, de Leon R, Roy RR, Zhong H, London NJ, Edgerton VR, Tillakaratne NJ (2009) Changes in $\mathrm{GABA}_{\mathrm{A}}$ receptor subunit gamma 2 in extensor and flexor motoneurons and astrocytes after spinal cord transection and motor training. Brain Res 1273:9-17.

Kiehn O (2006) Locomotor circuits in the mammalian spinal cord. Annu Rev Neurosci 29:279-306.

Kiehn O, Quinlan KA, Restrepo CE, Lundfald L, Borgius L, Talpalar AE, Endo $\mathrm{T}$ (2008) Excitatory components of the mammalian locomotor CPG. Brain Res Rev 57:56-63.

Kitzman P (2007) VGLUT1 and GLYT2 labeling of sacrocaudal motoneurons in the spinal cord injured spastic rat. Exp Neurol 204:195-204.

Krenz NR, Weaver LC (1998) Effect of spinal cord transection on N-methylD-aspartate receptors in the cord. J Neurotrauma 15:1027-1036.

Landry M, Bouali-Benazzouz R, El Mestikawy S, Ravassard P, Nagy F (2004) Expression of vesicular glutamate transporters in rat lumbar spinal cord, with a note on dorsal root ganglia. J Comp Neurol 468:380-394.

Lavrov I, Gerasimenko YP, Ichiyama RM, Courtine G, Zhong H, Roy RR, 
Edgerton VR (2006) Plasticity of spinal cord reflexes after a complete transection in adult rats: relationship to stepping ability. J Neurophysiol 96:1699-1710.

Macias M, Nowicka D, Czupryn A, Sulejczak D, Skup M, Skangiel-Kramska J, Czarkowska-Bauch J (2009) Exercise-induced motor improvement after complete spinal cord transection and its relation to expression of brain-derived neurotrophic factor and presynaptic markers. BMC Neurosci 10:144.

Magnusson T (1973) Effect of chronic transection on dopamine, noradrenaline and 5-hydroxytryptamine in the rat spinal cord. Naunyn Schmiedebergs Arch Pharmacol 278:13-22.

Murray KC, Nakae A, Stephens MJ, Rank M, D’Amico J, Harvey PJ, Li X, Harris RL, Ballou EW, Anelli R, Heckman CJ, Mashimo T, Vavrek R, Sanelli L, Gorassini MA, Bennett DJ, Fouad K (2010) Recovery of motoneuron and locomotor function after spinal cord injury depends on constitutive activity in 5-HT2C receptors. Nat Med 16:694-700.

Norreel JC, Pflieger JF, Pearlstein E, Simeoni-Alias J, Clarac F, Vinay L (2003) Reversible disorganization of the locomotor pattern after neonatal spinal cord transection in the rat. J Neurosci 23:1924-1932.

Norton JA, Mushahwar VK (2010) Afferent inputs to mid- and lowerlumbar spinal segments are necessary for stepping in spinal cats. Ann N Y Acad Sci 1198:10-20.

Petruska JC, Ichiyama RM, Jindrich DL, Crown ED, Tansey KE, Roy RR, Edgerton VR, Mendell LM (2007) Changes in motoneuron properties and synaptic inputs related to step training after spinal cord transection in rats. J Neurosci 27:4460-4471.

Plumier JC, Hopkins DA, Robertson HA, Currie RW (1997) Constitutive expression of the $27-\mathrm{kDa}$ heat shock protein (Hsp27) in sensory and motor neurons of the rat nervous system. J Comp Neurol 384:409-428.
Rank MM, Murray KC, Stephens MJ, D’Amico J, Gorassini MA, Bennett DJ (2011) Adrenergic receptors modulate motoneuron excitability, sensory synaptic transmission and muscle spasms after chronic spinal cord injury. J Neurophysiol 105:410-422.

Robinson GA, Goldberger ME (1986) The development and recovery of motor function in spinal cats. II. Pharmacological enhancement of recovery. Exp Brain Res 62:387-400.

Rossignol S, Brustein E, Bouyer L, Barthélemy D, Langlet C, Leblond H (2004) Adaptive changes of locomotion after central and peripheral lesions. Can J Physiol Pharmacol 82:617-627.

Rossignol S, Barrière G, Frigon A, Barthélemy D, Bouyer L, Provencher J, Leblond H, Bernard G (2008) Plasticity of locomotor sensorimotor interactions after peripheral and/or spinal lesions. Brain Res Rev 57:228-240.

Skup M, Wiater M, Górnicka E, Walentynowicz M, Czarkowska-Bauch J (2007) Different effect of locomotor exercise on the homogenate concentration of amino acids and monoamines in the rostral and caudal lumbar segments of the spinal cord in the rat. Spinal Cord 45:140-148.

Tillakaratne NJ, de Leon RD, Hoang TX, Roy RR, Edgerton VR, Tobin AJ (2002) Use-dependent modulation of inhibitory capacity in the feline lumbar spinal cord. J Neurosci 22:3130-3143.

Timoszyk WK, Nessler JA, Acosta C, Roy RR, Edgerton VR, Reinkensmeyer DJ, de Leon R (2005) Hindlimb loading determines stepping quantity and quality following spinal cord transection. Brain Res 1050:180-189.

Walwyn WM, Ta-Haung J, Ackerson L, Maidment NT, Edgerton VR (1999) Extracellular glutamate in the dorsal horn of the lumbar spinal cord in the freely moving rat during hindlimb stepping. Pharmacol Biochem Behav 63:581-588.

Weber ED, Stelzner DJ (1977) Behavioral effects of spinal cord transection in the developing rat. Brain Res 125:241-255. 